\title{
Treatment of uncomplicated gonorrhoea in women with a combination of rifampicin and erythromycin
}

\author{
A J BOAKES,* P S L LOO,* G L RIDGWAY,† S TOVEY, ₹ AND J D ORIEL* \\ From the Departments of *Genitourinary Medicine and + Microbiology, University College Hospital, and \\ the $¥$ Department of Genitourinary Medicine, the Middlesex Hospital, London
}

SUMMARY One hundred women with uncomplicated gonorrhoea (in five cases due to penicillinase producing strains of Neisseria gonorrhoeae (PPNG)) were treated with a single oral dose of rifampicin $900 \mathrm{mg}$ and erythromycin stearate $1 \mathrm{~g}$. $N$ gonorrhoeae was reisolated from the oropharynx of one patient, who was infected with a PPNG strain, but was eradicated from the genital tract in $100 \%$ of cases. The combination eradicated Chlamydia trachomatis from only 10 $(28 \%)$ of the 36 patients infected. Side effects were predominantly mild and consisted of transient nausea. The treatment merits evaluation in areas with a high incidence of PPNG strains.

\section{Introduction}

The emergence of penicillinase producing strains of Neisseria gonorrhoeae (PPNG) was predicted in $1975,{ }^{1}$ and outbreaks of clinical gonococcal infection caused by PPNG were identified shortly afterwards. ${ }^{23}$ The original foci of gonococcal infection with PPNG strains were in the Far East and West Africa, but since 1976 infection with PPNG strains has established itself as endemic in most parts of the world, and the increase in incidence has been exponential. ${ }^{4}$ The incidence of PPNG strains in some areas of South East Asia is now reported to be as high as $50 \%,{ }^{5}$ and a similar level has been reported in Nigeria (Osoba A O, unpublished data). In the United Kingdom the overall incidence of PPNG in 1982 was estimated at 2\%, but some sexually transmitted disease (STD) clinics in central London reported an incidence of more than $7 \%$, and abandoned the use of penicillin for the treatment of gonorrhoea in heterosexual patients. ${ }^{6}$ There is clearly an urgent need to evaluate treatment regimens that are alternatives to penicillin, and that are effective in a single dose, non-toxic and cheap. High cost is the most obvious disadvantage of some agents already evaluated, such as spectinomycin and "third generation" cephalosporins.

Despite the sensitivity of $N$ gonorrhoeae to erythromycin in vitro (minimum inhibitory concentration (MIC) about $0.06 \mathrm{mg} / \mathrm{l}) ;^{7}$ clinical results with the drug have been disappointing. Erythromycin

Address for reprints: Dr A J Boakes, Consultant Physician, Department of Genitourinary Medicine, University College Hospital, Gower Street, London WC1E 6AU

Accepted for publication 16 January 1984 base and estolate have given treatment failure rates approaching $25 \%,{ }^{8}$ and erythromycin stearate $1 \mathrm{~g}$ by mouth has given a failure rate of $28 \%$ (Jelinek, Oriel, Emmerson, and Ridgway, unpublished data). $N$ gonorrhoeae is also sensitive to rifampicin in vitro (MIC about $0.06 \mathrm{mg} / \mathrm{l}$ ). While a cure rate of $100 \%$ has been reported after a single dose of $1200 \mathrm{mg}$ rifampicin in the treatment of gonorrhoea, ${ }^{9}$ studies using $900 \mathrm{mg}$ have yielded much poorer results, with cure rates of $90 \%$ or less. ${ }^{10} 11$ Furthermore, when failure of treatment occurred there were pronounced differences between MICs before and after treatment, showing that treatment had resulted in the development of resistance. ${ }^{10}$ This phenomenon has also been shown in vitro. ${ }^{12}$ The addition of another antimicrobial agent (erythromycin or trimethoprim) to rifampicin in vitro, however, prevented the development of resistance to rifampicin. ${ }^{12} 13$ These findings prompted our evaluation of a combination of rifampicin and erythromycin in men with uncomplicated gonorrhoea, which resulted in a $96 \%$ cure rate ${ }^{14}$ Also impressive is the fact that the cost of the combination is one quarter to one fifth of recommended doses of cephalosporin derivatives that are stable to penicillinase. We now report on the treatment of women patients with the same combination.

\section{Patients and methods}

We studied 100 women patients at the departments of genitorurinary medicine of University College Hospital (76 patients) and the Middlesex Hospital (24 patients), London, between July 1982 and July 1983. 
Patients with confirmed or suspected pregnancy, known intolerance to rifampicin or erythromycin, or a history of antibacterial treatment in the preceding four weeks were excluded; otherwise patients were unselected. All patients gave informed verbal consent, and the protocol for the study was approved by local ethical committees.

A presumptive diagnosis of gonorrhoea was made if intracellular Gram negative diplococci were seen on microscopy, but this was confirmed by culture in every case. When possible, specimens were taken from the urethra, endocervix, rectum, and oropharynx for culture of $N$ gonorrhoeae on modified Thayer-Martin medium, but as the diagnosis was often unsuspected at the initial visit specimens were not obtained from all four sites in every case. In addition, material from the endocervix was transferred to transport medium for culture of Chlamydia trachomatis.

On entry to the study, patients were given (under supervision) rifampicin $900 \mathrm{mg}$ and erythromycin stearate $1 \mathrm{~g}$ orally with water. They were asked to return to the clinic three and 10 days after treatment for tests of cure, when the investigations described above were repeated.

\section{Results}

The diagnosis of gonorrhoea was confirmed by culture in all 100 patients, and all of them returned to the clinic for two sets of tests of cure. Their mean age was 24 (range 16-50) years and weight $58 \cdot 1 \mathrm{~kg}$ (range $44 \cdot 5-85 \mathrm{~kg}$ ); 88 were single, seven married, and five divorced; 83 were white and 17 black. At initial presentation $48 \%$ of patients had symptoms (usually. vaginal discharge or dysuria, or both) and $52 \%$ were symptomless; $62 \%$ were known contacts of men with gonorrhoea and $9 \%$ of cases occurred in symptomless women attending for routine check up.

The table gives details of the anatomical sites from which $N$ gonorrhoeae was isolated. All four sites were tested in 55 patients, $43(78 \%)$ of whom yielded positive results from the urethra, $50(91 \%)$ from the endocervix, $25(45 \%)$ from the rectum, and $5(9 \%)$ from the oropharynx. Overall, a presumptive diagnosis was made by microscopy in $67 \%$. Penicillin sensitivity tests yielded PPNG strains as the cause of

TABLE Isolation of $N$ gonorrhoeae from different anatomical sites of 100 women

\begin{tabular}{llll}
\hline Site & No tested & $\begin{array}{l}\text { No (\%) } \\
\text { positive }\end{array}$ & $\begin{array}{l}\text { No (\%) positive } \\
\text { at this site only }\end{array}$ \\
\hline Urethra & 100 & $76(76)$ & $5(5)$ \\
Endocervix & $99^{*}$ & $90(91)$ & $15(15)$ \\
Rectum & 89 & $39(44)$ & $4(4)$ \\
Oropharynx & 55 & $5(9)$ & \\
\hline
\end{tabular}

*One patient had had an hysterectomy infection in five patients who were known contacts of men infected with PPNG strains.

Sixty seven diagnoses of genitourinary infection other than gonorrhoea were made in 55 patients. These infections were due to $C$ trachomatis (36), Trichomonas vaginalis (14), Candida albicans (10), Gardnerella vaginalis (3), Escherichia coli (2), herpes simplex virus (1), and human papilloma virus (1).

All 100 patients returned for two tests of cure; the first at a mean of four (range two to 18) days after treatment, and the second at a mean of 12 (range seven to 28) days after treatment. $N$ gonorrhoeae was not isolated from any patient at the first follow up, but at the second was reisolated from one patient. In this case the reisolation was from the oropharynx, and was a PPNG strain, but the organism was not reisolated from either the urethra or endocervix, where it had been found initially.

$C$ trachomatis was isolated from 36 of the 100 patients. In $10(28 \%)$ the organism was isolated before but not after treatment, and in $15(42 \%)$ it was isolated both before and after treatment. In $11(30 \%)$ patients who all denied having further sexual intercourse, culture failed to isolate $C$ trachomatis before treatment but the organism was isolated after treatment. Candida albicans was isolated after treatment from high vaginal swabs from 28 patients whose swabs had been negative before treatment. Only seven of these patients had symptoms that required treatment with an antifungal agent.

Mild gastrointestinal symptoms (usually transient nausea occurring within a few hours of treatment) were reported by 28 patients, six reported vomiting, and a further five reported diarrhoea.

\section{Discussion}

Rifampicin has a wide range and high efficacy of antibacterial activity against both Gram positive and Gram negative micro-organisms. Fear has persisted, however, particularly in areas where there is high incidence of tuberculosis, that the general use of rifampicin may result in the emergence of resistant strains of mycobacteria. There is now good evidence that this fear is unfounded. Acocella et al, ${ }^{15}$ have compared the sensitivities of Mycobacterium tuberculosis in countries where rifampicin is reserved exclusively for mycobacterial infections with sensitivities in countries where the drug is used freely for all infections, and have found no difference between them. In addition, Grüneberg et al ${ }^{16}$ have shown that cultures of $\boldsymbol{M}$ tuberculosis exposed in vitro to therapeutic and sub-therapeutic levels of rifampicin for periods up to two weeks showed no change in their sensitivity to rifampicin. Thus there need be no fear that single doses, repeated single doses, or even 
short courses of rifampicin given for other infections, will impair in any way the efficacy of the drug in the treatment of mycobacterial infections.

Although rifampicin alone may be an effective treatment for gonorrhoea, the addition of another antimicrobial agent appreciably increases its efficacy by preventing the development of strains of $\mathrm{N}$ gonorrhoeae resistant to rifampicin. In this study a combination of rifampicin and erythromycin stearate proved to be highly effective, curing $99 \%$ of patients treated. The single treatment failure was in a patient infected with a PPNG strain that was reisolated from the oropharynx. Oropharyngeal gonorrhoea is generally regarded as being less vulnerable than genital infection to single dose treatment, and in this one treatment failure $\boldsymbol{N}$ gonorrhoeae was actually eradicated from the urethra and endocervix.

Both rifampicin and erythromycin are active against $C$ trachomatis in vitro. An earlier study of men with urethritis, however, showed that the combination was clinically ineffective against $C$ trachomatis, ${ }^{14}$ and this finding was confirmed in our study of women patients. Indeed it seems unlikely that any drug in a single dose will prove to be effective in the treatment of infection with $C$ trachomatis. We have previously seen that erythromycin stearate $500 \mathrm{mg}$ twice daily for seven days is an effective cure for $C$ trachomatis infections, ${ }^{17}$ and this regimen might usefully follow a single dose of rifampicin and erythromycin to eliminate concurrent chlamydial infection from patients with gonorrhoea.

We conclude that a combination of rifampicin and erythromycin stearate for the treatment of uncomplicated gonorrhoea is effective, safe, and cheap and merits further evaluation in areas where there is high incidence of PPNG strains.

\section{References}

1. Falkow S, Elwell LP, de Graaff J, Helfron F, Mayer L. A possible model for the development of plasmid-mediated penicillin resistance in the gonococcus. Conference on Sexually Transmitted Diseases. Royal Society of Medicine, London, 1975. London: Academic Press, 1976: 120-33.
2. Turner GC, Ratcliffe JG, Anderson D. Penicillinasc-producing Neisseria gonorrhoeae. Lancet 1976; ii: 793.

3. Golash RG, Hemming VG, Ashford WA, Moore FD, Waller TJ, Bettinger JJ, Presley CC. Penicillinase-producing Neisseria gonorrhoeae. Lancet 1976; ii: 793-4.

4. McCutchan JA, Adler MW, Berrie JRH. Penicillinase-producing Neisseria gonorrhoeae in Great Britain, 1977-81: alarming increase in incidence and recent development of endemic transmission. Br Med J 1982; 285:337-40.

5. Bloodworth LP, Daniels HA, Brockett RM, Tice AW. Rosaxacin in the treatment of Neisseria gonorrhoeae. South East Asian and Western Pacific Region Conference. Bangkok, Thailand: International Union against the Venereal Diseases and the Treponematoses, 1983 (in press).

6. McManus TJ, Harris JRW, Ison CA, Easmon CSF. Penicillinase-producing Neisseria gonorrhoeae. N Engl J Med 1982; 307: 1706.

7. Garrod LP, Lambert HP, O'Grady F. Antibiotics and chemotherapy. 5th ed. Edinburgh: Longman, 1981, 276-7.

8. Brown ST, Pederson AHR, Holmes KK. Comparison of erythromycin base and estolate in gonococcal urethritis. JAMA 1977; 238: 1371-3.

9. Soeltz-Szoets J. Today's therapy of gonorrhoea in female patients. 2nd Mediterranean Congress of Chemotherapy, Nice, France 1980 (in press).

10. Malmborg AS, Molin L, Nystrom B. Rifampicin compared with penicillin in the treatment of gonorrhoea. Chemotherapy 1971; 16:319-25.

11. Cobbold RJC, Marrison GD, Willcox RR. Treatment of gonorrhoea with single oral doses of rifampicin. Br Med $J$ 1968; ii:681-2.

12. Grüneberg RN, Emmerson AM, Felmingham D, Cremer $A W$. Ecological benefits of combining rifampicin with other antibiotics. In: Periti P, Grassi GG, eds. Proceedings of 12th International Congress of Chemotherapy, Florence, Italy. Washington DC: American Society for Microbiology, 1982; 2:253-4.

13. Ridgway GL, Grüneberg RN, Emmerson AM. Laboratory studies with erythromycin/rifampicin and doxycycline/DL473 combinations against Neisseria gonorrhoeae. In: Periti P, Grassi GG, eds. Proceedings of 12th International Congress of Chemotherapy, Florence, Italy. Washington DC: American Society for Microbiology, 1982;2:913-4.

14. Oriel JD, Ridgway GL, Goldmeier D, Felmingham D. Treatment of gonococcal urethritis in men with a rifampicin-erythromycin combination. Sex Transm Dis 1982;9:208-11.

15. Acocella G, Brumfitt W, Hamilton-Miller JMT. Evidence that rifampicin can be used safely for non-tuberculous diseases. Thorax 1980; 35: 788-91.

16. Grüneberg RN, Emmerson AM, Cremer AW. Improbability of selection of rifampicin resistance in mycobacterium tuberculosis by accidental exposure during short-course treatment. In: Periti P, Grassi GG, eds. Proceedings of the 12th International Congress of Chemotherapy, Florence, Italy. Washington DC: American Society for Microbiolgy, 1982; 2:991-2.

17. Oriel JD, Ridgway GL, Tchamouroff S. Comparison of erythromycin stearate and oxytetracycline in the treatment of non-gonococcal urethritis. Scott Med J 1977; 22:375-9. 